Table S12 Promoter cis element analysis and sequencing results.

\begin{tabular}{|c|c|c|c|}
\hline cis element & Core sequence & Function & Number \\
\hline ABRE & ACGTG & cis element involved in the abscisic acid responsiveness & 2 \\
\hline ARE & AAACCA & cis regulatory element essential for the anaerobic induction & 1 \\
\hline AT-rich sequence & TAAAATACT & element for maximal elicitor-mediated activation & 1 \\
\hline Box 4 & ATTAAT & part of a conserved DNA module involved in light responsiveness & 2 \\
\hline CAAT-box & CAAAT & common cis element in promoter and enhancer regions & 13 \\
\hline G-box & TACGTG & cis regulatory element involved in light responsiveness & 2 \\
\hline Pc-CMA2c & GCCCACGCA & part of a light responsive element & 1 \\
\hline TATA-box & TATATA & core promoter element around -30 of transcription start & 43 \\
\hline GCN4_motif & TGAGTCA & cis regulatory element involved in endosperm expression & 1 \\
\hline TCA-element & ССАТСТTТTТ & cis element involved in salicylic acid responsiveness & 1 \\
\hline cis element & Core sequence & Function & Number \\
\hline ARE & AAACCA & cis regulatory element essential for the anaerobic induction & 2 \\
\hline TATA-box & TATATA & core promoter element around -30 of transcription start & 5 \\
\hline Box 4 & ATTAAT & $\begin{array}{l}\text { part of a conserved DNA module involved in light } \\
\text { responsiveness }\end{array}$ & 9 \\
\hline CAAT-box & CAAAT & common cis element in promoter and enhancer regions & 15 \\
\hline CAT-box & GCCACT & cis regulatory element related to meristem expression & 1 \\
\hline G-box & CACGAC & cis regulatory element involved in light responsiveness & 1 \\
\hline
\end{tabular}

Analysis putative $c i s$ elements of the $V v U F G T$ promoter

Analysis putative cis elements of the $V v G S T 4$ promoter. 


\begin{tabular}{|c|c|c|c|}
\hline $\mathrm{O} 2$-site & GATGA(C/T)(A/G)TG(A/G) & $\begin{array}{l}\text { cis regulatory element involved in zein metabolism } \\
\text { regulation }\end{array}$ & 1 \\
\hline TCA-element & CCATCTTTTT & cis element involved in salicylic acid responsiveness & 1 \\
\hline GC-motif & CCCCCG & $\begin{array}{l}\text { enhancer-like element involved in anoxic specific } \\
\text { inducibility }\end{array}$ & 1 \\
\hline TATA-box & TATA & core promoter element around -30 of transcription start & 25 \\
\hline TCT-motif & TCTTAC & part of a light responsive element & 3 \\
\hline GT1-motif & GGTTAA & light responsive element & 1 \\
\hline
\end{tabular}

\section{Sequencing results of proVvGST4}

\section{ATG:}

TGCATAAAATGTTTAATTTTGTAAACATTTAAAAAAAAAAGAAATAAAAATAATAAGATA TAAGTTAAATCTATATGTTTTGTACAAAATGTTTTATTTTATATCATTTTTTAAATTTTTAAT ATAAATTTTTTTATTCAAAAAATAAAAAGTAGTAAGTAAATCCAAATAGATATTTAATGTT CGTATACTAATTTAAGTCTTTTTTCATTTTTATTTTTCATTTTCTCTTCTTTATTTTCTTTTTA TAATATTTTTTTTTCAAATCTTTTAAAAAGCAATGATATAGTTAAAATCTAATAATCCAAA TGATTATGGTTCATAAAAAGGCTTTTAAGCCCAATTGAATGTCAACCTGATGGATGGGTCA CCCAAATGATGATAGTCGAATGTCATACCATCGTGGTGGATGGGTCATCTAAATGATGGT AGTTTATAAAAAGGCTTTTAAACTTAGTTGAATGTAAGCCTAATGGATGGGCCACTTAAAT GGTGATAGTGCGATGTTATATCATCCCACTAAAGGGATCAACTAAATAATGGAGGTCAAT TGTTATAAAAAGGCTTATTGTACTCTCCTCAAATACTAATTAGTTTTTATATGAAATGATTA AAACCTGATTCAAGGTTAAAGATATGAGAAGAACTTATACCTTTTATAAGATATGTTTTAT TTTATACAAAGTTTTTAATTTAAAAAATAAAAAATAGTAAGTATATTTAAACTAATATTTA ATGTCTAGTTATTAATTTAAATTTATTTTTATTTTTCTTTTCTTTTCTTTTTATAATTTTTTTT TTTTTTAAATATGAGGGGAAAATGAAATGTCGTTGTGGGTGAACGTAAATGCATTATATTA AAAATCTCAATCTAGTAGATTTCATATATATCACTTAATAGTTATCTCACATATGGATTCC ATAAAATATTAAAGGAGGTTGTCCCTCTGGATATTGAATGAGTTTAAATAAATATATAAAT TAAAAATTATGTTGTAAAATAAGAATAAATGCAATATAAATAAAGTAGTATAAATAAAAT ATATCTTACTTTAAAAATATATATAATGGGAAGAATGAATTAAAGAAGAGAGTTGAGAAA GTTGAGAAAATGAGATAAAGTAAGAAATTTTTTTCTTACTTAGGGATGTATGTTACAGGG 
ATGGGAAACTCTTTTATAAGCTTTCTAAATAACTTCCATTAATATTCCTATTAATGAATATT AATGAAACTCATAAATAATATTAATGGTTTCTATTAATGAGTATTAATGGAAGTTATAAAT AATATTTAATTAACATTTATTATAATTAATATGGTGACATTCATTACTTTAATTATAACAAA TTATTAATTTAAATTTATTTTTCACTTTTTCCTGTATTTTCTTTTCTAATATTTTTCTTTAGAT TTTTTAAAAAGTAATGATAAGAGTTAAAAATGTCAAAACTGTAATAAATATGTGGAAAGA AATGAAGCATACAATGAATCTCCTCTAAAATCAGATCCAAGTTGATGAAAAGTGAAACCC AAGGTACCAGAAAGCGGAAAGCGTGCATGAATGTAACAATAATGTTTATAGTTATTGAAT GATCTTCTATAAATTGTGGTATTGAAGAAGGAATTTATTGTTGTCTGTTTTAAAAAGCCAC GATATCTTTTTTTGGTGGGTTTGACTATTTTTGGTGGGTTTGACACTTTTCCTCTCACCCAC CAGTAGATATTTGTATGTGACAACCCCCAAGAGGAAGAGGGACATGCAGCCTCTTTGAAA ATATTAAAATATGGAAGATTAGCAGAAAATATCAATATTTTTTTGTTAACTAATTACGTAA AACATGGGGATGAACCAAACTCATATGACAACCCCCGGATGGTTTGGACCGACAGCACCC TCAAATTTAGTGTTATAAAACAGGCGTTTTCTCCCCAGCTACACTCGACCAAACCTTAAAC AGCCAGATTATATATTGTGAAGAAGCAAGAGGTGGAAG

\section{CG:}

TGCATAAAATGTTTAATTTTGTAAACATTTAAAAAAAAAAGAAATAAAAATAATAAGATA TAAGTTAAATCTATATGTTTTGTACAAAATGTTTTATTTTATATCATTTTTTAAATTTTTAAT ATAAATTTTTTTATTCAAAAAATAAAAAGTAGTAAGTAAATCCAAATAGATATTTAATGTT CGTATACTAATTTAAGTCTTTTTTCATTTTTATTTTTCACTTTCTCTTCTTTATTTTCTTTTTA TAATATTTTTTTTTCAAATCTTTTAAAAAGCAATGATATAGTTAAAATCTAATAATCCAAA TGATTATGGTTCATAAAAAGGCTTTTAAGCCCAATTGAATGTCAACCTGATGGATGGGTCA CCCAAATGATGATAGTCGAATGTCATACCATCGTGGTGGATGGGTCATCTAAATGATGGT AGTTTATAAAAAGGCTTTTAAACTTAGTTGAATGTAAGCCTAATGGATGGGCCACTTAAAT GGTGATAGTGCGATGTTATATCATCCCACTAAAGGGATCAACCAAATAATGGAGGTCAAT TGTTATAAAAAGGCTTATTGTACTCTCCTCAAATACTAATTAGTTTTTATATGAAATGATTA AAACCTGATTCAAGGTTAAAGATATGAGAAGAACTTATACCTTTTATAAGATATGTTTTAT TTTATACAAAGTTTTTAATTTAAAAAATAAAAAATAGTAAGTATATTTAAACTAATATTTA ATGTCTAGTTATTAATTTAAATTTATTTTTATTTTTCTTTTCTTTTCTTTTTATAATTTTTTTT TTTTTCAAATATGAGGGGAAAATGAAATGTCGTTGTGGGTGAACGTAAATGCATTATATTA AAAATCTCAATCTAGTAGATTTCATATATATCACTTAATAGTTATCTCACATATGGATTCC ATAAAATATTAAAGGAGGTTGTCCCTCTGGATATTGAATGAGTTTAAATAAATATATAAAT TAAAAATTATGTTGTAAAACAAGAATAAATGCAACATAAATAAAGTAGTATAAATAAAAT ATATCTTACTTTAAAAATATATATAATGGGAAGAATGAATTAAAGAAGAGAGTTGAGAAA GTTGAGAAAATGAGATAAAGTAAGAAATTTTTTTCTTACTTAGGGATGTATGTTACAGGG ATGGGAAACTCTCTTATAAGCTTTCCAAATAACTTCCATTAATATTCCTATTAATGAATATT AATGAAACTCATAAATAATATTAATGGTTTCTATTAATGAGTATTAATGGAAGTTATAAAT 
AATATTTAATTAACATTTATTATAATTAATATGGTGACATTCATTACTTTAATTATAACAAA TTATTAATTTAAATTTATTTTTCACTTTTTCCTGTATTTTCTTTTCTAATATTTTTCTTTAGAT TTTTTAAAAAGTAATGATAAGAGTTAAAAATGTCAAAACTGTAATAAATATGTGGAAAGA AATGAAGCATACAATGAATCTCCTCTAAAATCAGATCCAAGTTGATGAAAAGTGAAACCC AAGGTACCAGAAAGCGGAAAGCGTGCATGAATGTAACAATAATGTTTACAGTTACTGAAT GATCTTCTATAAATTGTGGTATTGAAGAAGGAATTTATTGTTGTCTGTTTTAAAAAGCCAC GATATCTTTTTTTGGTGGGTTTGACTATTTTTGGTGGGTTTGACACTTTTCCTCTCACCCAC CAGTAGATATTTGTATGTGACAACCCCCAAGAGGAAGAGGGACATGCAGCCTCTTTGAAA ATATTAAAATATGGAAGATTAGCAGAAAATATCAATATTTTTTTGTTAACTAATTACGCAA AACATGGGGATGAACCAAACTCACATGACAACCCCCGGATGGTTTGGACCGACAGCACCC TCAAATTTAGTGTTATAAAACAGGCGTTTTCTCCCCAGCTACACTCGACCAAACCTTAAAC AGCCAGATTATATATTGTGAAGAAGCAAGAGGTGGAAG

Sequencing results of proVvUFGT

5ATG:

AAGTTAAACAAGGTGAGATGAGATGGCTATAGGAAATTCTCATACTTGGTTTATTTCGTCC CACCACATCTTGTTTAATTTTTTTAATGGGACGAGATAAAAATTATTTTGAACAAAAAAGG ATGGGATTGGGATAGAAGCCACCCATCCTAAACCCGCTCTGTTACCATCCTTACATAATAT CTAGGCATATAATATAAATCATGCACTCCAACACTATATATTGTATTTTTTTTAAAATCTA AACAACATAAAGTAAGAGCTCATGGAAGACATCATTAAAATATATTATTAAATTTAGGGT TAAATTATGGCATCTAAAATCAACTTTTTGGTATTATGTGTCCACCTATTATCAAATAGGA TATTGATATGTGTAAAAAAATAAATAAATAAATAAAAATCAAGATCTCATCTTGCACATC CAATGGTTGGTATGAATTCAATAATACCTATTAGCACAGTACTATAGAATTCTCCCTAAAT TTATGAAAAAATTACATTTTTGTAAGATCTATCTAACCTTTGATTCAGCACACTCTAGGGC AACATTGAGACAATTGTAGGTTGCAGCTTTTTAACATTCTCATTTTGCCCAAATATACAAA GCATGGAAACTAGAAAATATTGTGACTTGTTAAGGGCTTTTTTGTCATTTCATCTTGGTTTT GTTTGACATCATTTTTTTTTAAATTGTTTTTAAGAATAAAATTTATCTAGGAACATAAATAT GAAAAACGATATTTTGGTCTTATTCCATATGAGGGTGTTGTATAATTATATGCAATAAAAA AATTGTTGAAATATTTTTTATATTTCAATTGTTATCTAAAATACTTTACAAAAACACGATTG AGAGTATATTAGACTTGATCTTAAAAATAACTTGTTTTTAGAACATATTCTCAAAAAAATA TTTTCTGTAAAAAATTTAAGGTTTGTTTGATAACTGTTTTATAAAATAGTTATAAAAAATA GTTTTTTAAAACCATTATCTAATGTTTTGTAAAATAAAAATTTGTTTATAAATTTAAAATTT TTTAACATATTTTTAATATTTTTTAGTTTATTTTAAAAATAAGTTTGATATTTAGTATTTTAT TTTTAATCATTCTACATATTTGTATAATTATTTTTCAAAATATTCATAAAAAATAATTAAAA TATATTTTTTGAAAATACTTTGTTTTCTATTATAAATAATTTTTTATTGTTAAATGTATTATC 
TTAATTTTTTGTTTTAAAGAATAGAAAACTGTTCTAAAAAATAATTATCAAACCCTTACTTT TTAAGTTAAAATATAGTTTTTGGATCTTTTCAAAAAAGTTTTCAAACAACTCCTTTAATTTC TTTAATTAATTGCTTTTTCAAAATCAATTCTTAAAAAGTATTTTTTGAAAATAATTTTTTAA AATATTTTTTAATTGTTTTTGGTAACAAAATTCTATCTAATAACCAAAATATAAAAAATAA TTTTTTTAGTTCTCTTCATAAAAATAATATATATCATGTGGAATACAAAAATTTTTAAAAC GTTCTCCACCTTTTCAACATTCCTAAAAAAAAAAATTTTAAATTATTCTTAAAAATAATGG GGTTTTAATCAAATTAATTTTGAAAACATTAATTTATTTTAAAAAATTTATTGAATTATATT TTTCAAATTAGAAAACAATTTTATTTTCTTTAGAATGGAAAATAATTTTTTAAGTAAAATT AAAAAATATGCTCTTGCTACTACTCACTACGTATATCCCACCAATGGTAAAGTAAAAGTTC ACACAGAGCTTTCACTTGCCCTTGGTTTTGTTTTGTTTTTTTTCCCATTTTTTCTTTCTTTGG CCGCCATGTAGACTGGTGGTTTGGTTTTGGGTTGGTTTGTTAGGAGGGTGGGATGACAACC CCCATGCAGTTGCCACTCTCACAACCCCCATGCACTTGCTCTCATTTATAATCTTCAACAG CCAAAACCCAAATTGTAACATCCCCCATTCCAACC

\section{CG:}

AAGTTAAACAAGGTGAGACGAGATGGCTATAGGAAATTCTCATACTTGGTTTATTTCGTCC CACCACATCTTGTTTAATTTTTTTAATGGGACGAGATAAAAATTATTTTGAACAAAAAAGG ATGGGATTGGGATAGAAGCCACCCATCCTAAACCCGCTCTGTTACCATCCCTACATAATAT CTAGGCATATAATATAAATCATGCACTCCAACACTATATATTGTATTTTTTTTAAAATCTA AACAACATAAAGCAAGAGCTCATGGAAGACATCATTAAAATATATTATTAAATTTAGGGT TAAATTATGGCATCTAAAATCAACTTTTTGGTATTATGCGTCCACCTATTATCAAATAGGA TATTGATATGTGTAAAAAAATAAATAAATAAATAAAAATCAAGATCTCATCTTGCACATC CAATGGTTGGTATGAATTCAATAATACCTATTAGCACAGTACTATAGAATTCTCCCTAAAT TTATGAAAAAATTACATTTTTGTAAGATCTATCTAACCTTTGATTCAGCACACTCTAGGGC AACATTGAGACAATTGTAGGTTGCAGCTTTTCAACATTCTCATTTTGCCCAAATATACAAA GCATGGAAACTAGAAAATATTGTGACTTGTTAAGGGCTTTTTCGTCATTTCATCTTGGTTTT GTTTGACATCATTTTTTTTTAAATTGTTTTTAAGAATAAAATTTATCTAGGAACATAAATAT GAAAAACGATATTTTGGTCTTATTCCATATGAGGGTGTTGTATAATTATACGCAATAAAAA AATTGTTGAAATATTTTTTATATTTCAATTGTTATCTAAAATACTTTACAAAAACACGATTG AGAGTATATTAGACTTGATCTTAAAAATAACTTGTTTTTAGAACATATTCTCAAAAAAATA TTTTCTGTAAAAAATTTAAGGTTTGTTTGATAACTGTTTTATAAAACAGTTATAAAAAATA GTTTTTTAAAACCATTATCTAATGTTTTGTAAAATAAAAATTTGTTTATAAATTTAAAATTT TTTAACATATTTTTAATATTTTTTAGTTTATTTTAAAAATAAGTTTGATATTTAGTATTTTAT TTTTAATCATTCTACATATTTGTATAATTATTTTTCAAAACATTCATAAAAAATAATTAAAA TATATTTTTTGAAAATACTTTGTTTTCTATTATAAATAATTTTTTATTGTTAAATGTATTATC TTAATTTTTTGTTTTAAAGAATAGAAAACTGTTCTAAAAAATAATTATCAAACCCTTACTTT TTAAGTTAAAATATAGTTTTTGGATCTTTTCAAAAAAGTTTTCAAACAACTCCTTTAATTTC 
TTTAATTAATTGCTTTTTCAAAATCAATTCTTAAAAAGTATTTTTTGAAAATAATTTTTTAA AATATTTTTTAATTGTTTTTGGTAACAAAATTCTATCTAATAACCAAAATATAAAAAATAA TTTTTTTAGTTCTCTTCATAAAAATAATATATATCATGTGGAATACAAAAATTTTTAAAAC GTTCTCCACCTTTTCAACATTCCTAAAAAAAAAAATTTTAAATTATTCTTAAAAATAATGG GGTTTTAATCAAATTAATTTTGAAAACATTAATTTATTTTAAAAAATTTATTGAATTATATT TTTCAAATTAGAAAACAATTTTATTTTCTTTAGAATGGAAAATAATTTTTTAAGTAAAATT AAAAAATATGCTCTTGCTACTACTCACTACGTATATCCCACCAATGGTAAAGTAAAAGTTC ACACAGAGCTTTCACTTGCCCTTGGTTTTGTTTTGTTTTTTTTCCCATTTTTTCTTTCTTTGG CCGCCATGTAGACTGGTGGTTTGGTTTTGGGTTGGTTTGTTAGGAGGGTGGGATGACAACC CCCATGCAGTTGCCACTCTCACAACCCCCATGCACTTGCTCTCATTTATAATCTTCAACAG CCAAAACCCAAATTGTAACATCCCCCATTCCAACC 